\title{
Влияние типа и степени легирования на морфологию por-Si, полученного гальваническим травлением
}

\author{
(C) О.В. Пятилова ${ }^{1}$, С.А. Гаврилов ${ }^{1}$, Ю.И. Шиляева ${ }^{1}$, А.А. Павлов ${ }^{2}$, \\ Ю.П. Шаман ${ }^{3}$, А.А. Дудин ${ }^{2}$ \\ ${ }^{1}$ Национальный исследовательский университет „Московский институт электронной техники“, \\ 124498 Москва, Зеленоград, Россия \\ ${ }^{2}$ Институт нанотехнологий микроэлектрики Российской академии наук (ИНМЭ РАН), \\ 119991 Москва, Россия \\ ${ }^{3}$ Научно-производственный комплекс „Технологический центр“ МИЭТ, \\ 124498 Москва, Зеленоград, Россия \\ E-mail: 5ilova87@gmail.com
}

(Получена 17 мая 2016 г. Принята к печати 9 июня 2016 г.)

Изучен процесс формирования слоев пористого кремния гальваническим травлением в растворе $\mathrm{HF} / \mathrm{C}_{2} \mathrm{H}_{5} \mathrm{OH} / \mathrm{H}_{2} \mathrm{O}_{2}$ монокристаллического $\mathrm{Si}$, легированного бором и фосфором. Проведено исследование por-Si методами капиллярной конденсации азота и растровой электронной микроскопии. Установлены зависимости морфологических характеристик por- $\mathrm{Si}$, таких как диаметр пор, удельная площадь и объем поверхности, толщина стенок пор, определяющих кинетику горения $\mathrm{Si}$, от типа легирования и удельного сопротивления исходной пластины.

DOI: 10.21883/FTP.2017.02.44101.8327

\section{1. Введение}

Пористый кремний (por-Si), открытый в 1956 году [1], интенсивно изучается с середины 90-х гг. прошлого века благодаря широкому спектру применений, в том числе в качестве материала, быстрое окисление которого сопровождается горением или взрывом [2-4]. Как было показано в работах $[4,5]$, кинетика горения во многом определяется толщиной стенок пор, пористостью и удельной площадью поверхности por-Si. Чем больше площадь поверхности и тоньше стенки пор, тем выше скорость горения $\mathrm{Si}$. В этой связи определение закономерностей изменения морфологии пористого слоя от режимов формирования является важной задачей.

Наиболее часто por-Si формируют анодной обработкой в растворах плавиковой кислоты [6,7]. Изменение состава электролита, плотности тока, выбор типа и степени легирования $\mathrm{Si}$ позволяет изменять размеры пор в диапазоне от единиц нанометров до нескольких микрометров. Однако необходимость обеспечения однородного подвода тока к обрабатываемой поверхности существенно ограничивает возможности групповой обработки пластин. Более производительными и простыми с точки зрения аппаратурного исполнения при групповой обработке не только пластин, но и порошков, являются методы, не требующие применения внешнего источника тока. К ним относятся stain etching [8-10], локальное химическое травление кремния с использованием металла ( $\mathrm{Ag}[11], \mathrm{Au}[12], \mathrm{Pt}[13]$ и т.д.) в качестве катализатора (metal-assisted chemical etching - MACE) и гальваническое травление (galvanic etching) [14,15]. Последний из указанных методов является предметом исследования настоящей работы.
Для обеспечения гальванического травления обрабатываемый материал частично покрывают пленкой металла, который является катодом по отношению к реакции растворения кремния. Нанесение металла осуществляют либо вакуумным испарением [16], либо из раствора, содержащего ионы металла, которые могут самопроизвольно восстанавливаться на поверхности кремния, например ионы $\mathrm{Ag}^{+}$[17]. На покрытой металлом поверхности в растворах, содержащих НF и сильные окислители, такие как $\mathrm{H}_{2} \mathrm{O}_{2}$ [18], $\mathrm{VO}_{2}^{+}$[19] и $\mathrm{Cr}_{2} \mathrm{O}_{7}^{2-}[20]$, в результате гальванического травления кремния на поверхности образуются микро-, мезо- и макропористые слои. Морфология получаемых слоев зависит от режимов обработки, таких как состав электролита, отношение площадей контакта металла и кремния с электролитом [3], тип металла [21], длительность травления [22]. В работе [3] показано, что снижение концентрации HF в электролите приводит к росту диаметра пор, пористости и удельной поверхности por-Si. Также пористость растет с увеличением длительности обработки [22].

С точки зрения применения таких слоев в качестве энергетических материалов остается не изученной важная задача определения влияния технологических параметров травления на толщину стенок пор. Известно, что кинетика горения $\mathrm{Si}$ зависит от толщины слоя и пористости [4], а также от состава окислителя [5]. Очевидно, что и толщина стенок пор может существенно влиять на скорость горения.

Цель настоящей работы - исследование влияния типа проводимости и удельного сопротивления $\mathrm{Si}$ на свойства получаемых пористых слоев, в частности на удельную площадь поверхности, диаметр и толщину стенок пор, и, как следствие, на кинетику горения. 

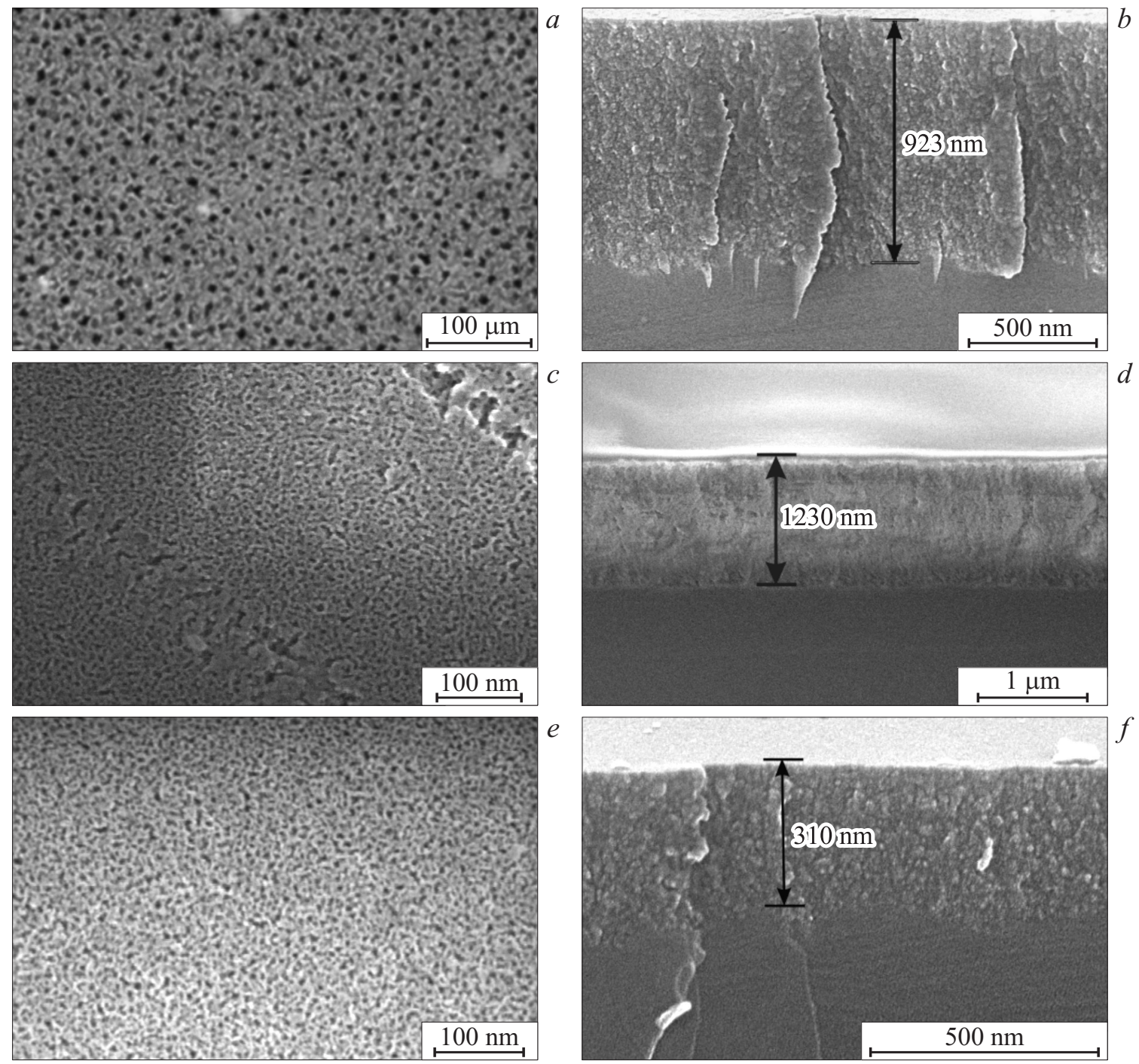

Рис. 1. РЭМ-изображения поверхности и скола por- $\mathrm{Si}$, сформированного в растворе $\mathrm{HF} / \mathrm{C}_{2} \mathrm{H}_{5} \mathrm{OH} / \mathrm{H}_{2} \mathrm{O}_{2}$ на пластинах $p$-типа $(\rho=0.01$ Ом $\cdot$ см $)-(a, b) ; p$-типа $(\rho=1$ Ом $\cdot$ см $)-(c, d), n$-типа $(\rho=4.5$ Ом $\cdot$ см $)-(e, f)$.

\section{2. Методика эксперимента}

Пористые слои формировали на поверхности пластин монокристаллического кремния $p$-типа проводимости $(p-\mathrm{Si})$ с удельным сопротивлением $\rho=0.01$ и $1 \mathrm{OM} \cdot \mathrm{cm}$, $n$-типа проводимости $(n-\mathrm{Si})$ с удельным сопротивлением $\rho=4.5 \mathrm{OM} \cdot$ см с ориентацией поверхности (100). Перед нанесением металла пластины обрабатывали в течение 10 мин при температуре $130^{\circ} \mathrm{C}$ в растворе Каро, содержащем $\mathrm{H}_{2} \mathrm{SO}_{4} \quad(98 \%): \mathrm{H}_{2} \mathrm{O}_{2} \quad(30 \%)$ в объемном соотношении $1: 1$. Далее на нерабочую сторону пластин магнетронным распылением последовательно наносили пленки Ті и $\mathrm{Pt}$ толщиной 15 и 80 нм соответственно. Пленку Тi, не контактирующую с электролитом, использовали для улучшения адгезии благородного металла к кремнию.

Формирование пористых слоев проводили при освещенности 460 Лк в смеси водных растворов HF (40\%) и $\mathrm{C}_{2} \mathrm{H}_{5} \mathrm{OH}(90 \%)$ в объемном соотношении (3:1) с добавкой $\mathrm{H}_{2} \mathrm{O}_{2}(30 \%)$, количество которой составляло $2.5 \%$ от общего объема электролита при температуре $T=25^{\circ} \mathrm{C}$ [3]. Раствор $\mathrm{HF} / \mathrm{C}_{2} \mathrm{H}_{5} \mathrm{OH} / \mathrm{H}_{2} \mathrm{O}_{2}$ широко применяется в технологии создания пористого кремния гальваническим травлением [23]. Для практического применения пористого кремния в качестве энергетического материала толщина пористого слоя оказывает большое влияние на скорость горения [4], поэтому, исходя из экспериментальных данных, минимальное время обработки кремниевой пластины было выбрано равным 10 мин. Длительность процесса варьировали в диапазоне $t=10-40$ мин. После обработки образцы промывали в водном растворе этилового спирта для удаления электролита, продуктов реакции травления и сушили на воздухе.

Исследования морфологии пористого кремния проводили с помощью растровой электронной микроскопии 

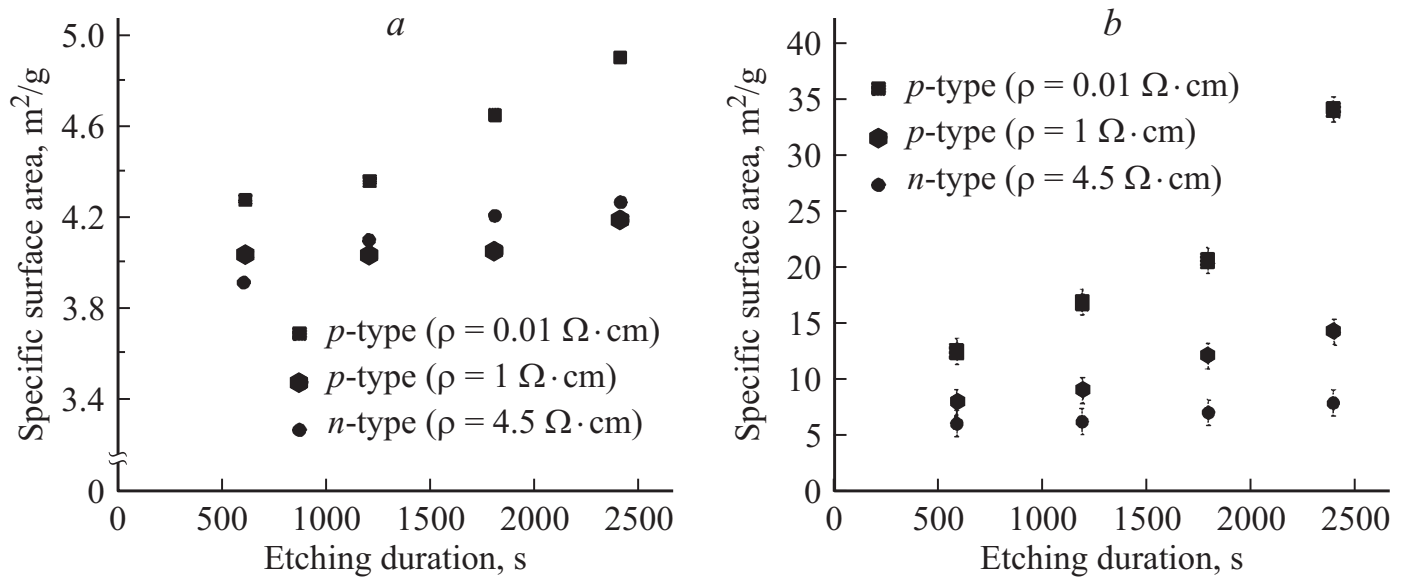

Рис. 2. Зависимости среднего диаметра пор $(a)$ и площади поверхности пористых слоев $(b)$, полученных на кремнии с разной степенью и типом легирования, от длительности травления.

(микроскоп Helios NanoLab 650). Удельную площадь поверхности, пористость и размеры пор оценивали с помощью сорбтометра Quantachrome NOVA 3200e.

Дополнительно для подтверждения возможности практического применения пористого кремния в качестве энергетического материала образцы пропитывали перхлоратом натрия окунанием в $3.2 \mathrm{M}$ раствор $\mathrm{NaClO}_{4}$ в $\mathrm{C}_{2} \mathrm{H}_{5} \mathrm{OH}$ при ультразвуковой агитации в течение 1 мин. Далее образцы сушили при $T=60^{\circ} \mathrm{C}$, горение инициировали нагреванием до $T=350^{\circ} \mathrm{C}$.

\section{3. Результаты}

На рис. 1 представлены РЭМ-изображения por-Si, сформированных в течение 10 мин на пластинах с различным удельным сопротивлением и типом легирования.

Как следует из полученных результатов микроскопии скорость формирования слоя por-Si на кремнии $p$-типа в несколько раз выше, чем на кремнии $n$-типа при одинаковых режимах обработки (рис. $1 b, d, f)$. Эти результаты согласуются с известными представлениями об образовании пористого кремния, согласно которым скорость растворения Si в HF зависит от концентрации дырок в полупроводнике [24].

Для определения удельной площади поверхности, толщины стенок и диаметра пор в объеме слоя por-Si был проведен анализ удельной поверхности образцов методом капиллярной конденсации азота [25]. Изотермы адсорбции-десорбции всех исследуемых образцов имели вид, характерный для мезопористых материалов. Для всех исследованных образцов в диапазоне давлений $p / p_{0}$ от 0.05 до 0.35 изотермы адсорбции были линейными. Это позволило рассчитать удельную площадь поверхности пористых слоев исследуемых структур, используя многоточечную модель БЭТ (Брунауэр, Эммет и Теллер) [26]. Полученные зависимости среднего диаметра пор и удельной площади поверхности пористых слоев с разной степенью и типом легирования от длительности обработки представлены на рис. 2.

Из зависимостей на рис. 2 можно сделать вывод, что слои пористого кремния, сформированные на пластине кремния $p$-типа проводимости с $\rho=0.01 \mathrm{OM} \cdot \mathrm{cm}$, обладают максимальным значением диаметра пор и удельной площади поверхности по сравнению с por-Si на пластине $p$-типа проводимости с $\rho=1$ Ом · см и $n$-типа проводимости с $\rho=4.5 \mathrm{OM} \cdot \mathrm{cm}$.

Зная суммарный удельный объем и средний диаметр пор, толщину пористого слоя, а также допустив в первом приближении цилиндрическую форму пор, можно рассчитать среднее расстояние между осями пор $D$ для por-Si с разной степенью и типом легирования:

$$
D=\sqrt{\frac{S_{s u r} h_{p o r} d^{2}}{V_{p o r}}},
$$

где $V_{\text {por }}$ - измеренный методом сорбтометрии суммарный удельный объем пор пористого слоя, $S_{s u r}-$

Морфологические характеристики пористых слоев

\begin{tabular}{|c|c|c|c|}
\hline Образец & $\begin{array}{c}\text { Длительность } \\
\text { обработки, мин }\end{array}$ & $V_{p o r}, \mathrm{~cm}^{3} / \Gamma$ & $a, \mathrm{HM}$ \\
\hline$p$-Si c $\rho=0.01 \mathrm{OM} \cdot \mathrm{cm}$ & $\begin{array}{l}10 \\
20 \\
30 \\
40\end{array}$ & $\begin{array}{l}0.018618 \\
0.025461 \\
0.031797 \\
0.054214\end{array}$ & $\begin{array}{l}8.1 \\
6.7 \\
6.3 \\
4.4\end{array}$ \\
\hline$p-\mathrm{Si} \mathrm{c} \rho=1 \mathrm{OM} \cdot \mathrm{cm}$ & $\begin{array}{l}10 \\
20 \\
30 \\
40\end{array}$ & $\begin{array}{l}0.007435 \\
0.009128 \\
0.009586 \\
0.013816\end{array}$ & $\begin{array}{r}13.3 \\
11.9 \\
11.4 \\
9.6\end{array}$ \\
\hline$n-\mathrm{Si} \mathrm{c} \rho=4.5 \mathrm{OM} \cdot \mathrm{cm}$ & $\begin{array}{l}10 \\
20 \\
30 \\
40\end{array}$ & $\begin{array}{l}0.005939 \\
0.007009 \\
0.007413 \\
0.008162\end{array}$ & $\begin{array}{l}14.7 \\
14.0 \\
13.9 \\
13.4\end{array}$ \\
\hline
\end{tabular}


$a$
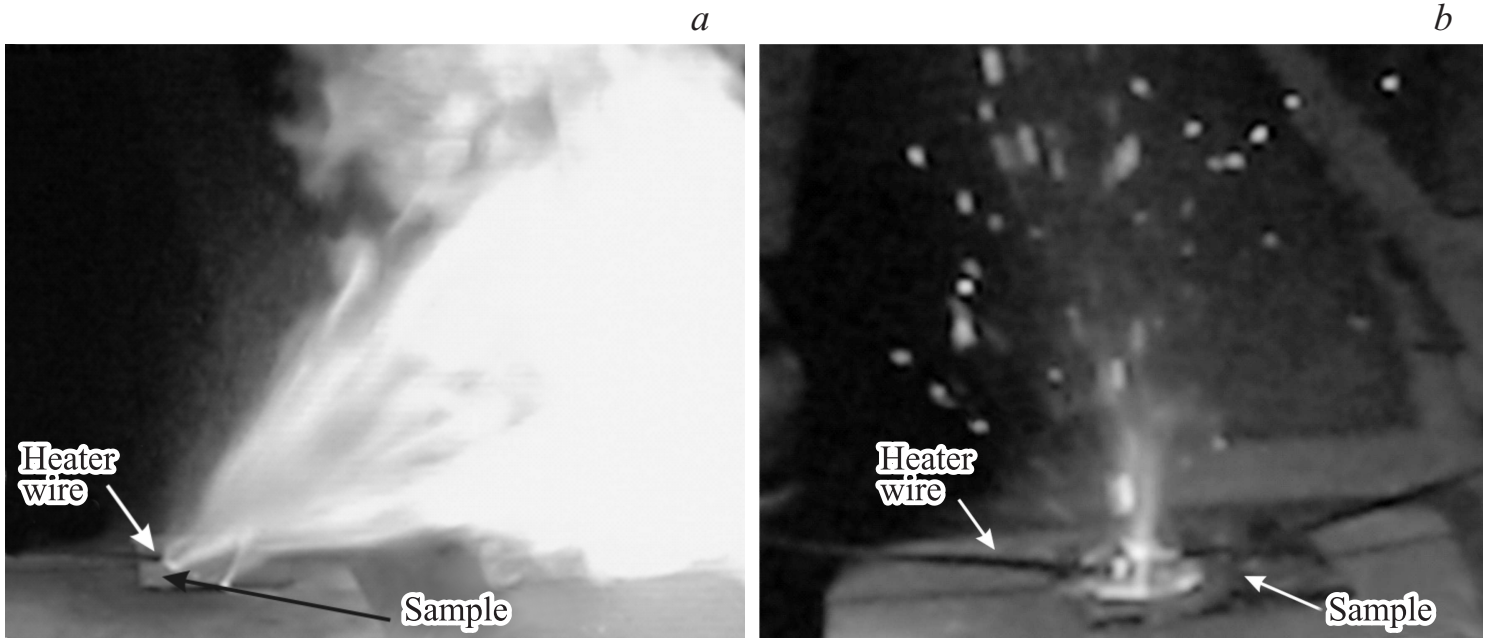

Рис. 3. Фотографии процесса горения пористого кремния, сформированного при травлении длительностью 30 мин на $p$-Si c $\rho=0.01 \mathrm{OM} \cdot \mathrm{cm}(a)$ и $p$-Si с $\rho=1 \mathrm{OM} \cdot \mathrm{cm}(b)$.

площадь поверхности образца до травления, $h_{p o r}-$ толщина пористого слоя (из результатов РЭМ), $d$ - измеренный методом сорбтометрии внутренний диаметр пор.

Зная $D$, можно рассчитать толщину стенки пор $a$, которая определяется выражением

$$
a=\frac{(D-d)}{2}
$$

В таблице представлены измеренные и рассчитанные по формулам (1)-(2) морфологические характеристики por-Si, сформированного на различных подложках при различной длительности травления.

Показано, что минимальную толщину стенок имеют пористые слои на $p$-Si c $\rho=0.01 \mathrm{OM} \cdot$ см. Толщина стенок пор на $p$-Si c $\rho=1$ Ом - см выше, чем у $p$-Si с $\rho=0.01 \mathrm{OM} \cdot \mathrm{cm}$, в 1.64 и 2.2 раза при длительности обработки 10 и 40 мин соответственно. Толщина стенок пор на $n$-Si с $\rho=4.5 \mathrm{OM} \cdot$ см выше, чем у $p$-Si с $\rho=0.01 \mathrm{OM} \cdot \mathrm{cm}$, в 1.81 и 3 раза при длительности обработки 10 и 40 мин соответственно. Обнаруженная зависимость толщины стенок пор от удельного сопротивления кремния тривиально объясняется тем, что растворение $\mathrm{Si}$ требует непрерывной доставки дырок к растворяемой поверхности.

Экспериментально установлено, что скорость горения пористого $p$-Si c $\rho=0.01$ и 1 Ом - см тем выше, чем больше длительность обработки в $\mathrm{HF} / \mathrm{C}_{2} \mathrm{H}_{5} \mathrm{OH} / \mathrm{H}_{2} \mathrm{O}_{2}$. Это может быть обусловлено уменьшением толщины стенок пор, увеличением удельной площади поверхности и толщины пористого слоя. Процесс горения не наблюдается у por-Si, полученного обработкой длительностью до 20 мин $p$-Si с $\rho=1$ Ом - см, а у por- $\mathrm{Si}$, полученного на $n-\mathrm{Si}$, - даже после 40 мин травления. На рис. 3 представлены фотографии процесса горения пористого кремния $p$-Si, пропитанного спиртовым раствором перхлората натрия.
Таким образом, в работе были получены экспериментальные зависимости удельной площади поверхности, суммарного удельного объема, диаметра и толщины стенок пор от длительности обработки в растворе $\mathrm{HF} / \mathrm{C}_{2} \mathrm{H}_{5} \mathrm{OH} / \mathrm{H}_{2} \mathrm{O}_{2}$ для $\mathrm{Si}$ с разным типом и степенью легирования. Установлено, что пористый $p$-Si c $\rho=0.01$ Ом · см обладает максимальным значением диаметра пор, удельной площади поверхности и минимальным значением толщины стенок пор по сравнению с $p$-Si с $\rho=1$ Ом · см и $n$-Si с $\rho=4.5$ Ом · см. Установлено, что интенсивность горения por-Si растет с уменьшением $a$, следовательно, будет максимальной для $p$-Si c удельным сопротивлением менее 1 Ом · см.

Работа выполнена при финансовой поддержке Российского научного фонда (проект № 16-19-10625).

\section{Список литературы}

[1] A. Uhlir. Bell Syst. Techn., 35, (2), 333 (1956).

[2] D. Kovalev, V.Y. Timoshenko, N. Kunzner, E. Gross, F. Koch. Phys. Rev. Lett., 87, 301 (2001).

[3] C.R. Becker, S. Apperson, Ch.J. Morris, Sh. Gangopadhyay, L.J. Currano, W.A. Churaman, C.R. Stoldt. Nano Lett., 11, 803 (2011).

[4] С.К. Лазарук, А.В. Долбик, П.В. Жагиро, В.А. Лабунов, В.Е. Борисенко. ФТП, 39 (8), 917 (2005).

[5] M. du Plessis. Sensors Actuators A, 135, 666 (2007).

[6] D.R. Turner. J Electrochem. Soc., 105, 402 (1957).

[7] V. Lehmann, U. Gösele. Appl. Phys. Lett., 58, 856 (1991).

[8] S. Liu, C. Palsule, S. Yi, S. Gangopadhyay. Phys. Rev. B, 49 (15), 10318 (1994).

[9] J. Xu, A.J. Steck. J. Vac. Sci. Technol. B, 13 (3), 121 (1995).

[10] R.W. Fathauer, T. George, A. Ksendzov, R.P. Vasquez. Appl. Phys. Lett. 60 (8), 995 (1992).

[11] Y. Qu, L. Liao, Yu. Li, H. Zhang, Yu Huang, X. Duan. Nano Lett., 9 (12), 4539 (2009). 
[12] Ch.-L. Lee, K. Tsujino, Yu. Kanda, Sh. Ikeda, M. Matsumura. J. Mater. Chem., 18, 1015 (2008).

[13] Z. Huang, N. Geyer, P. Werner, J. De Boor, U. Gösele. Advanced Mater., 23 (2) 285 (2011).

[14] C.M.A. Ashruf, P.J. French, P.M.M.C. Bressers, P.M. Sarro, J.J. Kelly. Sensors Actuators A: Physical, 66 (1-3), 284 (1998).

[15] K.W. Kolasinski. Nanoscale Res. Lett., 9, 432 (2014).

[16] T. Hadjersi, N. Gabouze, N. Yamamoto, K. Sakamaki, H. Takai, A. Ababou, E.S. Kooij. Phys. Status Solidi C, 2 (9), 3384 (2005).

[17] O.V. Pyatilova, S.A. Gavrilov, A.A. Dronov, Ya.S. Grishina, A.N. Belov. Sol. St. Phenomena, 213, 103 (2014).

[18] D. Wang, R. Ji, S. Du, A. Albrecht, P. Schaaf. Nanoscale Res. Lett., 8, 42 (2013).

[19] K.W. Kolasinski, W.B. Barclay, Y. Sun, M. Aindow. Electrochim. Acta., 158, 219 (2015).

[20] T. Hadjersi, N. Gabouze. Optical Mater., 30, 865 (2008).

[21] X. Li, P.W. Bonn. Appl. Phys. Lett., 77 (16), 2572 (2000).

[22] M. Lipinskia, J. Cichoszewski, R.P. Socha, T. Piotrowski. Acta Phys. Polon. A, 116, 117 (2009).

[23] Д.Н. Горячев, Л.В. Беляков, О.М. Сресели. ФТП, 37 (4), 494 (2003).

[24] V. Parkhutik. Sol. St. Electron., 43 (6), 1121 (1999).

[25] E.P. Barret, L.G. Joyner, P.P. Halenda. J. Amer. Chem. Soc., 73, 373 (1951)

[26] А.С. Вячеславов, Е.А. Померанцева, Е.А. Гудилин. Методическая разработка: Измерение площади поверхности $и$ пористости методом капиллярной конденсации азота (М., МГУ, 2006) с. 55.

Редактор Г.А, Оганесян

\section{Influence of the type and doping level on the morphology of porous silicon produced by galvanic etching}

O.V. Pyatilova1, S.A. Gavrilov'1, Yu.I. Shilyaeva', A.A. Pavlov', Yu.P. Shaman ${ }^{3}$, A.A. Dudin ${ }^{2}$

${ }^{1}$ National Research University of Electronic Technology, 124498 Moscow, Zelenograd, Russia

${ }^{2}$ Institute of Nanotechnology in Microelectronics

Russian Academy of Science (INME RAS),

119991 Moscow, Russia

${ }^{3}$ Scientific-Manufacturing Complex

„Technological Centre“ MIET

(SMC „Technological Centre"),

124498 Moscow, Zelenograd, Russia

Abstract In this paper, formation porous silicon by galvanic etching of single cryst wafers in $\mathrm{HF}+\mathrm{C}_{2} \mathrm{H}_{5} \mathrm{OH}+\mathrm{H}_{2} \mathrm{O}_{2}$ solution has been investigated. The porous layers have been studied using capillary condensation of nitrogen and scanning electron microscopy. Dependence of morphology characteristics of porous silicon on doping type and resistivity of the etched wafer has been defined. It was shown additionally that morphology characteristics of the porous layers, such as pore diameters, specific surface area, cumulative volume of pores and thickness of pore walls, have great influence on kinetics of porous silicon combustion. 